Randomized Trial

\title{
C-Arm Guided Percutaneous Radiofrequency Thoracic Sympathectomy for Treatment of Primary Palmar Hyperhidrosis in Comparison with Local Botulinum Toxin Type A Injection, Randomized Trial
}

Tarek A.H. Mostafa, MS1', Abdel A. Hamed, MD', Basma M. Mohammed, MD², Nabil A. El Sheikh, MD', and Ahmed A.A. Shama, MD ${ }^{1}$

From: ${ }^{1}$ Anesthesia, Intensive Care and Pain Medicine Department, Faculty of Medicine, Tanta University, Egypt; '2Dermatology and Venerology Department,

Faculty of Medicine, Tanta University, Egypt

Address Correspondence: Tarek A.H. Mostafa, MS Department of Anesthesia and

Surgical Intensive Care

Faculty of Medicine, Tanta University

El-Geish St.

Tanta, Egypt 31257 E-Mail:

dr.tarek311@yahoo.com

Disclaimer: There was no external funding in the preparation of this manuscript.

Conflict of interest: Each author certifies that he or she, or a member of his or her immediate family, has no commercial association

(i.e., consultancies,

stock ownership, equity

interest, patent/licensing arrangements, etc.) that might

pose a conflict of interest in connection with the submitted manuscript.

Manuscript received: 02-01-2019

Revised manuscript received: 04-25-2019

Accepted for publication: 06-18-2019

Free full manuscript: www.painphysicianjournal. com
Background: Hyperhidrosis is a disorder associated with detrimental effects on patients' quality of life, occupational activities, and social interactions.

Objectives: This study compares $\mathrm{C}$-arm guided percutaneous radiofrequency (RF) ablation of the second and third thoracic sympathetic ganglions and local intradermal botulinum toxin type A (BTX-A) injection for the treatment of primary palmar hyperhidrosis. It focuses on clinical effectiveness, patient satisfaction, quality of life, safety, and the time at which repetition of the procedure is needed over one-year follow-up.

Study Design: This is a randomized single-blinded trial.

Setting: This study took place in a single hospital.

Methods: Eighty patients with primary palmar hyperhidrosis were randomly assigned to one of 2 interventions: local intradermal BTX-A injection $(n=40)$ or $C$-arm guided percutaneous RF ablation ( $n$ = 40). The Dermatology Life Quality Index (DLQI) questionnaire and the Hyperhidrosis Disease Severity Scale (HDSS) were used for assessment at one week, one month, and 2, 6, and 12 months after intervention. The number of patients who required repetition of the procedure later on and the time at which they needed it were recorded, and possible side effects were assessed.

Results: HDSS scores in the RF group were statistically significantly lower than in the BTX-A group at one week, one month, and 2, 6, and 12 months of follow-up. DLQI scores in the RF group were statistically significantly lower than in the BTX-A group at 6- and 12-month follow-up, whereas at one week, one month, and 2 months of follow-up, there was no statistically significant difference between both groups. The number of patients who required that the procedure be repeated was statistically significantly lower in the RF group than in the BTX-A group. The time at which patients needed repetition of the procedure in the BTX-A group was about 3 to 7 months after the first intervention. All patients in this group showed an increase in HDSS scores within this one-year followup. In the RF group, however, only one patient complained of increased HDSS scores after 8 months. There was no statistically significant difference in side effects between both groups.

Limitations: The first limitation of this study is that results were based on subjective scales. The second is the radiation exposure associated with the technique described.

Conclusions: This study supports percutaneous C-arm guided RF ablation of the second and third thoracic sympathetic ganglions and local intradermal BTX-A injection as safe, effective options and rapid lines of treatment of primary palmar hyperhidrosis. However, percutaneous RF ablation proved to be more effective, with longer effectiveness time and better patient satisfaction, compared to local intradermal BTX-A injection.

Key words: Botulinum toxin, hyperhidrosis, quality, radiofrequency ablation

Pain Physician 2019: 22:591-599 
yperhidrosis is defined as excessive sweating beyond what is necessary for the maintenance of thermal regulation, without any known etiology such as rise in temperature or mental stimuli. It greatly affects the patient's quality of life and their ability to perform occupational activities and to interact (1). It is a complex disorder of the autonomic nervous system involving the sympathetic and parasympathetic pathways. Palmar hyperhidrosis is considered to be a neurologic disorder with exuberant cutaneous manifestation (2). It is widely classified into 2 types: primary and secondary (3).

Between $60 \%$ and $80 \%$ of patients with primary hyperhidrosis have a family history with a pattern of inheritance suggesting autosomal dominant transmission with incomplete penetrance (4).

Botulinum toxin is a natural protein product of Clostridium botulinum bacteria. It prevents the release of acetylcholine at the neuromuscular junction of striated muscle fibers, resulting in flaccid paralysis of the muscle (5). Botulinum toxin, however, effectively treats both palmar and axillary hyperhidrosis, as well as certain types of headaches (6).

Several techniques have been proposed to execute thoracic sympathectomy, including surgical intervention, percutaneous sympathetic neurolysis, and radiofrequency (RF) ablation, which is the least invasive technique using selector magnetic energy near the nerve tissue (7).

The purpose of this study was to compare C-arm guided percutaneous RF ablation of the second and third thoracic sympathetic ganglions and local intradermal botulinum toxin type A (BTX-A) injection for the treatment of primary palmar hyperhidrosis. Factors being compared include clinical effectiveness, patient satisfaction, quality of life, safety, and time at which repetition of the procedure is needed.

\section{Methods}

\section{Study Design and Patients}

This randomized trial was performed in the pain management unit and dermatology department at Tanta University Hospitals between December 2015 and March 2018. The purpose, benefits, and potential risks of the study were explained to the patients, and each asked to sign a written informed consent. Every patient had a secret code number, and photographs only of the related body part were captured and stored to ensure their privacy and data confidentiality.
Procedures were approved by both the institutional and regional ethical committees (approval number: 30512/09/15). CONSORT guidelines were followed in reporting.

Patients enrolled in the study were those diagnosed with primary palmar hyperhidrosis, including excessive sweating for at least 6 months with no obvious cause. In addition, they had to be displaying at least 2 of the following features: impairment of daily activities, a bilateral and relatively symmetric pattern of sweating occurring at least once per week, age older than 16 years, cessation of focal sweating during sleep, or positive family history.

Exclusion criteria included patient's refusal or those who were uncooperative, primary palmar hyperhidrosis with previous surgical intervention, secondary hyperhidrosis, neuromuscular diseases such as myasthenia gravis, being administered drugs such as aminoglycosides that interfere with neuromuscular activity, pregnancy or lactation, infection, skin disease, keloid formation at the surgery site, implantation of a pacemaker or defibrillator that magnetic waves may interfere with, and coagulopathy (international normalized ratio $\geq 1.5$, platelets count $\leq 80.000$ ).

\section{Randomization and Blinding}

Eighty patients were randomly assigned to either the local intradermal BTX-A injection group (BTX-A group: 40 patients) or the C-arm guided percutaneous RF ablation group (RF group: 40 patients). An independent data manager assigned the patients to groups based on a computer-generated randomization program. The patients and the outcome assessor, who was a physician independent from the outpatient pain management clinic, were blinded to each patient's randomization number. All procedures were performed by a single provider.

\section{Intervention}

For each patient, intravenous (IV) access was secured using an 18-gauge cannula. In addition, an electrocardiogram, a noninvasive blood pressure monitor, and a pulse oximeter were attached.

In the BTX-A group, skin of the palms was prepared with a mixture of lidocaine $2.5 \%$ and prilocaine $2.5 \%$ cream (EMLA 5\% cream, AstraZeneca, Cambridge, United Kingdom) at least one hour before injection. A $100 \mathrm{lU} /$ powder vial of BTX-A (BOTOX, Allergan Inc., Madison, NJ) was reconstituted with $5 \mathrm{~mL}$ sterile normal saline solution $0.9 \%$ to obtain 2 units $/ 0.1 \mathrm{~mL}$ re- 
constitution, and was used immediately within 4 hours using a 27-gauge needle (insulin needle) for injection. The skin of the palm was sterilized before injection. The desired injection site was the deep dermis at or near the dermal-subcutaneous junction, where the sweat glands are located. It was important not to inject too deep as this may cause muscle weakness, especially over the thenar eminence, where the subcutaneous and dermis layers are thin, leading to decreased manual skills and grip strength.

Injection caused bleeding and extrusion of BTXA. To minimize drug loss, the injection was made at an oblique angle, about $45^{\circ}$, with the bevel facing upward to create a longer injection track. The needle was advanced in until a slight decrease in resistance was felt on entering the dermis. With each injection, a small amount of blanching was expected as the drug was correctly injected intradermally. The total dose and number of injection sites varied based on the size of the patient's hand. Each injection was composed of $0.1 \mathrm{~mL}$ ( 2 units of BTX-A). The average dose was $100 \mathrm{U}$ BTX-A per palm, ranging from 80 to 200 .

The injection grid pattern was as follows: 2 or 3 sites were injected in the distal phalanx of the hand as it may be a problematic sweating area and is in constant use. Two sites were injected in both the middle and proximal phalanges. About 15 to 20 sites were injected approximately 1 to $1.5 \mathrm{~cm}$ apart (as the radial diffusion distance of BTX-A was about 1.0 to $1.5 \mathrm{~cm}$ ); each row contained about 4 injection sites. Injections at the fingertips were performed superficially because of the presence of an extensive capillary network. The dominant writing hand received a row of injections beginning at the base of the little finger and continuing to the wrist, midway between the palm and dorsal surface (medial edge of the hand), to provide maximum dryness when writing.

Following injection, light pressure was applied to the treated area for a few seconds to aid in hemostasis and decrease chances of bruising. Even distribution and migration of the injected BTX-A within the locally treated area was also an added benefit of applying firm pressure. The patients were instructed to close their fists tightly, occasionally for 1 to 2 hours to increase presynaptic uptake of the toxin, while avoiding massaging of the treated area to prevent bruising. Clinical effects generally began to appear within 2 to 3 days of injection. The nondominant hand was first injected for feedback and dose adjustment, and then the dominant hand was treated based on that 2 weeks later. Each patient was monitored for 2 hours after the procedure for potential complications, and then they were immediately discharged.

In the RF group, the patients were placed in the prone decubitus position, arms elevated, head in neutral position, and a pillow under the chest on a fluoroscopic-compatible table to flex the thoracolumbar spine. A nasal cannula with $5 \mathrm{~L} /$ minute flow rate of $\mathrm{O}_{2}$ was attached to each patient. They received $2 \mathrm{mg}$ of midazolam IV and/or nalbuphine $(10 \mathrm{mg}$ ) if needed for sedation and analgesia. The patients were awake, cooperative, and responsive to the physician's questions after needle placements.

The C-arm (Ziehm Vision R, Ziehm Imaging Inc., Nuremberg, Germany) was first placed in the anteroposterior position to visualize the C7, T1, T2, and T3 vertebral bodies. It was rotated obliquely, approximately $20^{\circ}$ toward the ipsilateral side. It was then moved in a cephalocaudal direction approximately $20^{\circ}$ to open the intervertebral space of the $T 2$ vertebral body and to "square it up" following 2 radiologic landmarks, specifically the tubercle of the costotransverse articulation and the costovertebral articulation (head of the rib), to achieve a comparatively straightforward passage (tunnel view) to the thoracic ganglia. The purpose of this was to reduce the risk of pneumothorax. The needle was advanced in via the "direction-depthdirection" technique, with the aid of a fluoroscope to confirm correct position of the needle.

The tip of the triangle formed by the T2 vertebral body and the third rib was the target point. Forceps were used to identify the point of skin entrance at the lateral edge of the T2 vertebral body cephalad to the third rib (2.5 inches). The skin was anesthetized using a 25 -gauge needle ( $3 \mathrm{~cm}$ needle) with $2 \%$ lidocaine. The skin at the site of entry was completely sterilized before the insertion of the needle.

Using a curved 10-mm active tip RF needle under fluoroscopic guidance with the posterior-anterior and lateral view, with a tilt in the cephalocaudal direction, the tunnel view to the target area was maintained deep into the tissues. The needle was advanced in under tunneled vision through the entry point toward the target, until it touched the vertebral body. Throughout, the needle was always kept in close contact with the lateral edge of the $\mathrm{T} 2$ vertebral body. The $\mathrm{C}$-arm was positioned laterally, and the needle was advanced until it reached the junction between the anterior two-thirds and the posterior one-third of the T2 vertebral body. The C-arm was positioned again in the posterior-anterior position to confirm that the needle was advanced correctly. Two 
milliliters of contrast medium solution was injected to confirm the position of the needle under fluoroscopic guidance after aspiration test. The dye spread up and down the thoracic vertebral column to confirm that the tip of the RF needle was away from motor and sensory intercostal nerves, discs, and the thecal sac. The thermocouple of the RF machine (NeuroTherm NT2000 IX, St. Judge Medical, Abbot, St. Paul, MN) was inserted through the RF needle, measuring tissue impedance on motor and sensory stimulation.

Two needles were inserted at the second and third thoracic sympathetic ganglions at the same time. Two milliliters of lidocaine $2 \%$ was injected to decrease the pain produced by RF lesions before the beginning of ablation. At the end, the patient was observed for at least 2 hours. Staged RF therapy was applied unilaterally. The other side was treated 2 weeks later to avoid the risk of bilateral pneumothorax and compensatory hyperhidrosis due to sympathetic overflow.

\section{Outcome Assessment and Follow-Up}

The baseline characteristics of all included patients were gathered. The primary outcome of the study was clinical effectiveness assessed by the Hyperhidrosis Disease Severity Scale (HDSS), in relation to patient satisfaction and quality of life reviewed by the Dermatology Life Quality Index (DLQI) questionnaire. Secondary outcomes included patient safety with the least side effects, and the time at which repetition of the procedure was needed. Cases were assessed before the procedure and one week, one month, and 2, 6, and 12 months after the procedure.

\section{Statistical Analysis}

SPSS Version 24 (IBM Corporation, Armonk, NY) was used for data processing and statistical analysis. Sample size was calculated at $\mathrm{n} \geq 38$ for each study group based on $95 \%$ confidence limit and $80 \%$ power of the study. The ratio between the 2 study groups was $1: 1$. The expected outcome ranged between $55 \%$ and $85 \%$ of the ideal outcome.

Categorical variables were presented as absolute numbers and percentages. Continuous variables were presented as mean values with standard deviation or medians with an interquartile range. To compare data between groups, the chi-square test was used to assess categorical variables, and the Student test or the Mann-Whitney $U$ test was used to analyze continuous variables as appropriate. Paired t test or the Wilcoxon test was used for comparison within the same group as appropriate. A 2-tailed $P$ value of $<0.05$ was considered to indicate a statistically significant difference.

\section{ResUlts}

For this clinical study, 88 patients were thought to be eligible. However, 8 were excluded either because they did not fulfill the inclusion criteria (5 patients: 3 had previous surgical intervention for hyperhidrosis, one patient was pregnant, and one had an infection at the site of technique application) or because they refused to participate (3 patients). The remaining 80 patients were randomly allocated into 2 equal groups. Data of all patients were successfully collected (Fig. 1).

Demographic data, including age and gender, were comparable between the 2 groups $(P=0.0991$ for age; $P=0.654$ for gender).

In the BTX-A group, there was a statistically significant decrease in HDSS scores at one week, one month, and 2 months after treatment, compared with scores before treatment $(P=0.0001,0.0001$, and 0.0001 , respectively). The scores at 6 and 12 months after treatment were not significantly different from before treatment ( $P=0.18$ and 0.058 , respectively) (Table 1 ).

In the RF group, there was a statistically significant decrease in HDSS scores at one week, one month, and 2,6 , and 12 months after treatment, compared with scores before treatment $(P=0.0001,0.0001,0.0001$, 0.0001 , and 0.0001 , respectively) (Table 2 ).

Comparison between both groups regarding HDSS scores showed a statistically significant difference at one week, one month, and 2, 6, and 12 months after treatment (scores in the RF group were significantly lower than in the BTX-A group, as $P=0.002,0.001$, $0.002,0.0001$, and 0.0001, respectively) (Fig. 2).

Regarding DLQI, in the BTX-A group, there was a statistically significant decrease in scores at one week, one month, and 2 and 6 months after treatment, compared with scores before treatment $(P=0.0001$, $0.0001,0.0001$, and 0.001 , respectively). The scores at 12 months after treatment were not significantly different from before treatment $(P=0.808)$ (Table 3$)$. In the RF group, there was a statistically significant decrease in DLQI scores at one week, one month, and 2,6, and 12 months after treatment, compared with scores before treatment $(P=0.0001,0.0001,0.0001,0.0001$, and 0.0001 , respectively) (Table 4).

Comparing the 2 groups regarding DLQI showed a statistically significant difference at 6 and 12 months after treatment (the RF group showed scores statistically significantly lower than the BTX-A group, as $P=$ 


\section{Enrollment}

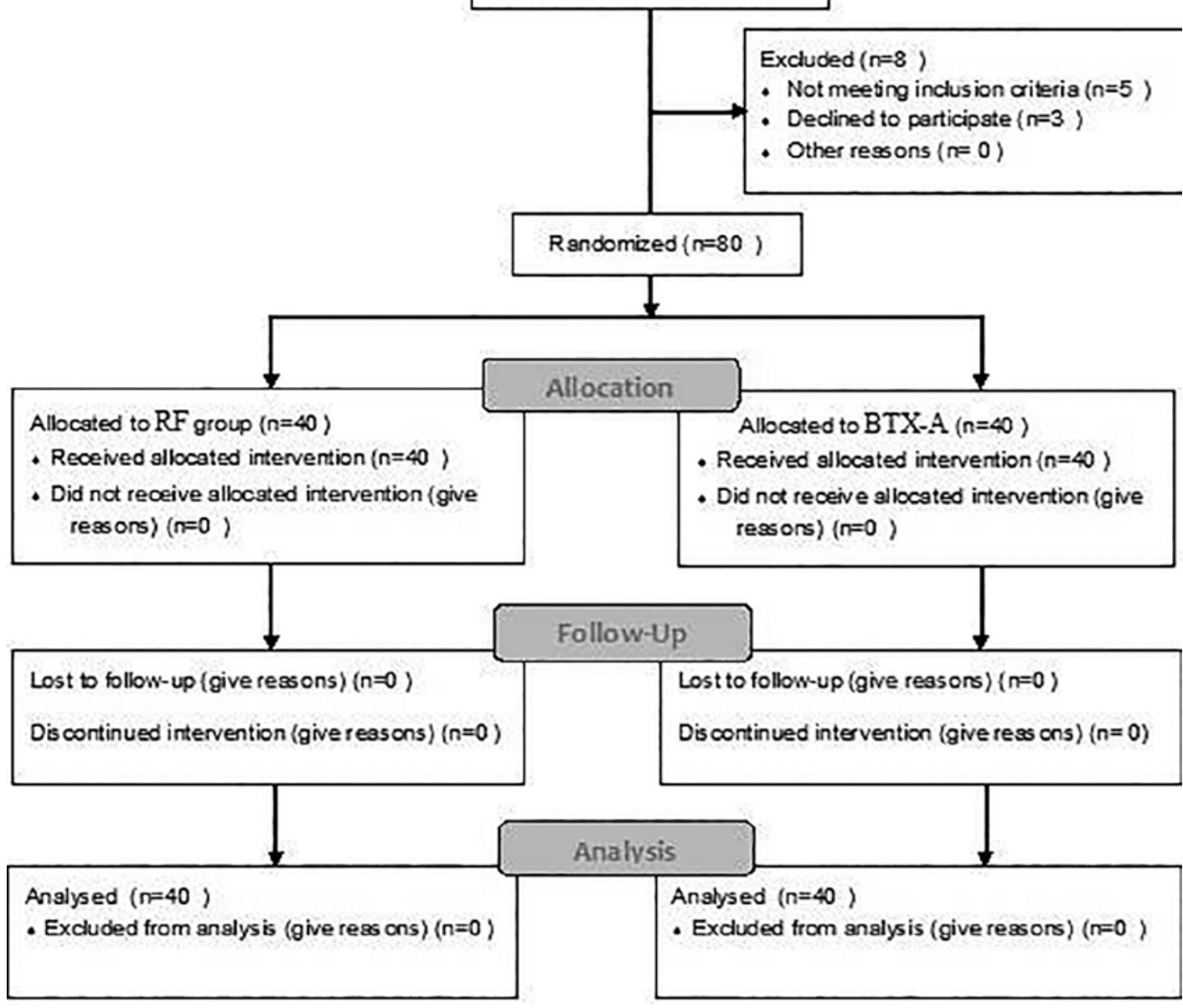

Fig. 1. CONSORT flow chart.

Table 1. HDSS of BTX-A group.

\begin{tabular}{|l|c|c|c|c|c|c|}
\hline \multicolumn{7}{|c|}{ HDSS of BTX-A group } \\
\hline & \multirow{2}{*}{$\begin{array}{c}\text { Before } \\
\text { treatment }\end{array}$} & \multicolumn{5}{|c|}{ After treatment } \\
\cline { 3 - 8 } & & $\mathbf{1}$ week & $\mathbf{1}$ month & $\mathbf{2}$ months & $\mathbf{6}$ months & 12 months \\
\hline Median & 3 & 2 & 1.5 & 1.5 & 3 & 3 \\
(Q1-Q3) & $(2-3)$ & $(1-2)$ & $(1-2)$ & $(1-2)$ & $(2-3)$ & $(2.25-3.75)$ \\
\hline Wilcoxon test & $P$ value & 0.0001 & 0.0001 & 0.0001 & 0.18 & 0.058 \\
\hline \hline
\end{tabular}

Table 2. HDSS of RF group.

\begin{tabular}{|l|c|c|c|c|c|c|}
\hline \multicolumn{9}{|c|}{ HDSS of RF group } \\
\hline & \multirow{2}{*}{$\begin{array}{c}\text { Before } \\
\text { treatment }\end{array}$} & \multicolumn{7}{c|}{ After treatment } \\
\cline { 3 - 7 } & & 1 week & 1 month & 2 months & 6 months & 12 months \\
\hline $\begin{array}{l}\text { Median } \\
\text { (Q1-Q3) }\end{array}$ & $\begin{array}{c}1 \\
(2-3)\end{array}$ & $(1-1)$ & $\begin{array}{c}1 \\
(1-0)\end{array}$ & $\begin{array}{c}1 \\
(1-1)\end{array}$ & $(1-1)$ & 1 \\
\hline Wilcoxon test & $P$ value & 0.0001 & 0.0001 & 0.0001 & 0.0001 & 0.0001 \\
\hline
\end{tabular}




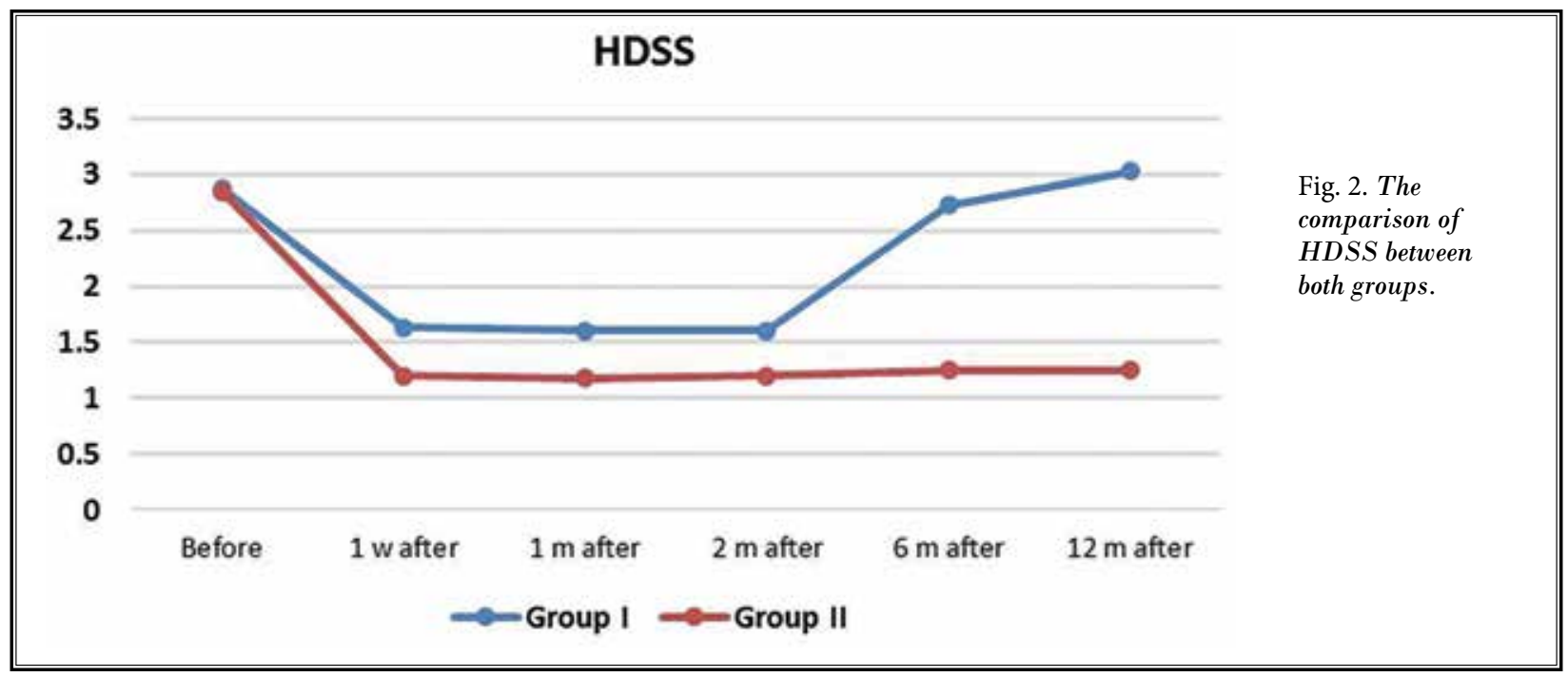

Table 3. DLQI score of BTX-A group.

\begin{tabular}{|l|c|c|c|c|c|c||}
\hline \multicolumn{7}{|c|}{ DLQ1 score of BTX-A group } \\
\hline & \multirow{2}{*}{$\begin{array}{c}\text { Before } \\
\text { treatment }\end{array}$} & \multicolumn{5}{|c|}{ After treatment } \\
\cline { 3 - 7 } & 14.5 & 1 week & 1 month & 2 months & 6 months & 12 months \\
\hline $\begin{array}{l}\text { Median } \\
\text { Q1-Q3) }\end{array}$ & $(8-18)$ & $(.025-4)$ & $\begin{array}{c}1 \\
(0-4)\end{array}$ & $\begin{array}{c}1 \\
(0-4)\end{array}$ & $\begin{array}{c}1 \\
(6-14)\end{array}$ \\
\hline Wilcoxon test & $P$ value & 0.0001 & 0.0001 & 0.0001 & 0.0001 & 0.808 \\
\hline
\end{tabular}

Table 4. DLQI score of RF group.

\begin{tabular}{|l|c|c|c|c|c|c|}
\hline \multicolumn{1}{|c|}{ DLQ1 score of RF group } \\
\hline & \multirow{2}{*}{$\begin{array}{c}\text { Before } \\
\text { treatment }\end{array}$} & \multicolumn{7}{|c|}{ After treatment } \\
\cline { 3 - 7 } & 13 & 1 week & 1 month & 2 months & 6 months & 12 months \\
\hline $\begin{array}{l}\text { Median } \\
\text { (Q1-Q3) }\end{array}$ & 1 & 1 & 1 & 1 & 1 \\
$(13-24.9)$ & $(1-3)$ & $(1-3)$ & $(1-3)$ & $(1-3.9)$ & $(1-3.9)$ \\
\hline Wilcoxon test & $P$ value & 0.0001 & 0.0001 & 0.0001 & 0.0001 & 0.0001 \\
\hline
\end{tabular}

0.0001 and 0.0001 , respectively). However, there was no statistically significant difference between before treatment and one week, one month, and 2 months after treatment $(P=0.691,0.165,0.165$, and 0.207 , respectively) (Fig. 3).

The number of patients who required that the procedure be repeated in the RF group was statistically significantly lower than in the BTX-A group $(P=0.00001)$. The time at which repetition of the procedure was needed in the BTX-A group was approximately 3 to 7 months after intervention, as all patients in this group complained of increased HDSS scores within one-year follow-up. However, in the RF group, only one patient complained from an increased HDSS score after 8 months, within one-year follow-up (Table 5).

In the BTX-A group, only 9 (22.5\%) patients experienced side effects: one patient $(2.5 \%)$ experienced tenderness at the site of the procedure, and 8 patients $(20 \%)$ experienced warmth and dryness of the skin of the palm. In the RF group, 12 patients (30\%) experienced side effects: 3 patients $(7.5 \%)$ showed compensatory axillary hyperhidrosis, and 9 patients $(22.5 \%)$ experienced warmth and dryness of the skin of the palm. Therefore, there was no statistically significant difference between both groups $(P=0.65)$. 
Fig. 3. The comparison of DLQI scores between both groups.

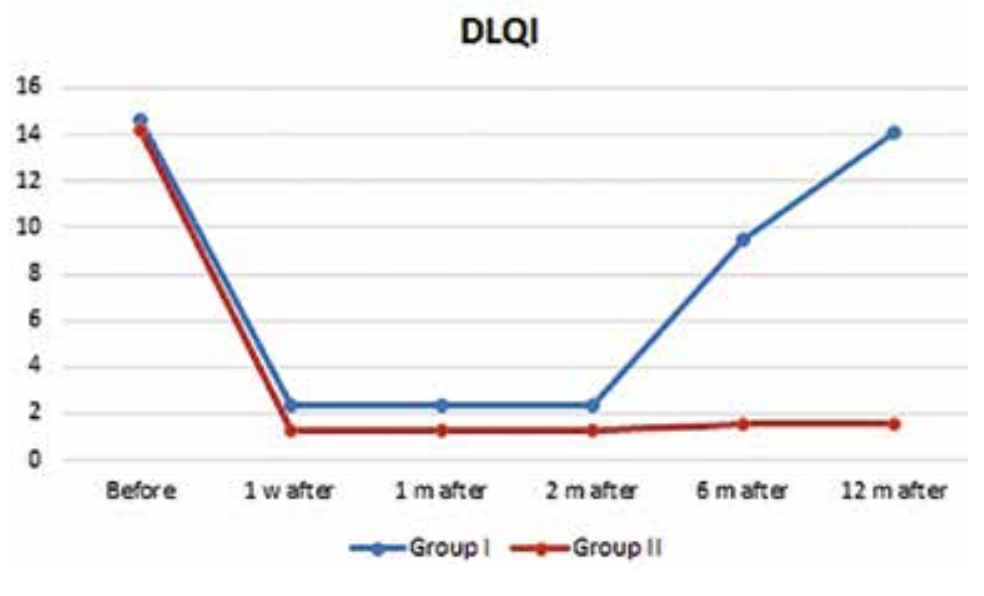

\section{Discussion}

This study assessed clinical effectiveness, patient satisfaction, quality of life, safety, and time at which repetition of the procedure was needed in C-arm guided percutaneous RF ablation of the second and third thoracic sympathetic ganglions as a line of treatment for primary palmar hyperhidrosis versus local intradermal BTX-A injection.

To the authors' knowledge, this is the first study to compare both techniques for treatment of primary hyperhidrosis. HDSS scores in the RF group were statistically significantly lower than in the BTX-A group at one week, one month, and 2, 6, and 12 months of follow-up. However, the DLQI scores in the RF group were statistically significantly lower than in the BTX-A group at 6 and 12 months of follow-up. The number of patients who needed repetition of the procedure was statistically significantly lower in the RF group than in the BTX-A group. The time at which repetition of the procedure was needed in the BTX-A group was about 3 to 7 months after treatment. All patients in this group complained of increased HDSS scores within one-year follow-up, whereas in the RF group, only one patient complained of that after 8 months. No statistically significant difference was found in side effects between both groups.

Many studies were done by Romero et al (8). They compared different therapeutic methods, such as the use of topical drugs, systemic drugs, iontophoresis, local botulinum injection, and finally sympathectomy by endoscopic or percutaneous RF. They found that videoassisted thoracoscopic sympathectomy was the most effective treatment for palmar hyperhidrosis, with long-
Table 5. The number of patients needed for repeating the procedure.

\begin{tabular}{|l|c|c||}
\hline \hline \multicolumn{2}{|c|}{ Number of Patients Needed for Repeating the Procedure } \\
\hline & Number & $\%$ \\
\hline Group I & 40 & $100 \%$ \\
\hline Group II & 1 & $2.5 \%$ \\
\hline \multirow{2}{*}{ Chi-square test } & $\mathrm{X} 2$ & $P$ value \\
\cline { 2 - 3 } & 2.225 & 0.00001 \\
\hline
\end{tabular}

lasting functional results. However, the percutaneous procedure was conducted under local anesthesia with minimal invasiveness, one-day hospitalization, rapid recovery, low morbidity, zero mortality, and early return to daily activities (8).

In a study conducted in 2018, Romero et al (9) reported that upper thoracic percutaneous sympathectomy with RF positively affected the quality of life for patients with palmar hyperhidrosis.

Also, a prospective study by Garcia Franco et al (10) on percutaneous RF sympathicolysis for the treatment of severe palmar hyperhidrosis revealed that surgery was more effective $(P=0.0001)$ and led to better quality of life $(P=0.0002)$ than RF sympathicolysis. However, $70.9 \%$ of patients were dissatisfied as they experienced back pain immediately after the RF procedure, and some patients reported numbness extending to the axillary area, which disappeared within one week after treatment. Patients who were treated with RF did not complain of compensatory sweating, unlike most patients who were treated surgically and reported mild to moderate compensatory sweating. Nevertheless, patients with complete remission of hyperhidrosis after 
RF procedure experienced a mild form of compensatory sweating. Such a complaint was owing to the complete ablation of both chains, similar to what happened in surgically treated patients (10).

The current study was also in agreement with the Purtuloglu et al (11) study that compared RF to surgical sympathectomy. RF successfully treated $75 \%$ of patients with hyperhidrosis, which is statistically lower than the surgical sympathectomy success rate $(P<0.01)$. However, patient satisfaction and occurrence of compensatory hyperhidrosis were similar in both groups $(P=0.26$ and $P=0.78$, respectively). The incidence of compensatory hyperhidrosis was $35 \%$ in both groups, which is lower than the previous study, which could be attributed to the selection of lower levels for sympathectomy (11).

García-Barquín et al (12) evaluated the benefits of computed tomography-guided percutaneous sympathicolysis with RF in patients with primary palmar hyperhidrosis. They reported improvement of at least one point on the HDSS in $77.38 \%$ of hands after one month following the procedure, and $59.52 \%$ at longterm follow-up. Statistically significant worsening in HDSS scores $(P<0.05)$ was observed when treatment efficacies at one month and in long-term follow-up were compared. Only 2 major complications were observed as unsatisfactory in $52.38 \%$ of the patients, and $1.8 \%$ developed either massive pneumothorax or anaphylaxis to opioids. Three patients reported minor complications $(2.1 \%)$. Two patients complained of severe pain at the injection site during the procedure, and one patient developed small pneumothorax that did not require chest tube placement (12).
Naumann and Lowe $(13)$ and Lowe et al $(14,15)$ in their studies used Botox for axillary hyperhidrosis with a follow-up period of 12 months. They reported a time range between treatments (duration of effect) of 15.4 to 51.3 weeks (mean: 30.6 weeks), with $80 \%$ mean reduction in sweat production after the second treatment.

Studies conducted by Heckmann et al (16) and Lowe et al (17) reported $75 \%$ reduction in sweating, improvement in emotional and physical well-being, and reduced limitations of daily activity, without any serious adverse events in cases refractory to topical antiperspirants on botulinum toxin injection.

There are several limitations to this study. The main limitation is that the results are based on subjective scales. The second is radiation exposure associated with the technique, which necessitates precautions to reduce it. Moreover, the inability to precisely display the sympathetic system may have led to missing some anatomic variations and accessory fibers, such as the Kuntz nerve.

\section{Conclusions}

This study presents both percutaneous C-arm guided RF ablation of the second and third thoracic sympathetic ganglions and local intradermal BTX-A injection as promising modalities of treatment of primary palmar hyperhidrosis. However, percutaneous RF ablation proved to be more effective, exhibited the longest effectiveness time, and provided the best level of patient satisfaction, compared to local intradermal BTX-A injection. 


\section{References}

1. Walling HW. Clinical differentiation of primary from secondary hyperhidrosis. J Am Acad Dermatol 2011; 64:690-695.

2. Birner P, Heinzl $H$, Schindl M, Pumprla J, Schnider P. Cardiac autonomic function in patients suffering from primary focal hyperhidrosis. Eur Neurol 2000; 44:112-116.

3. Miller J, Hurley $\mathrm{H}$. Diseases of the eccrine and apocrine sweat glands. In: Bolognia JL, Jorizzo JL, Rapini RP (eds). Dermatology. 2nd ed. Spain, Mosby Elsevier, 2008: pp. 567-588.

4. Higashimoto I, Yoshiura Ki, Hirakawa N, et al. Primary palmar hyperhidrosis locus maps to 14q11.2-q13. Am J Med Genet A 2006; 140:567-572.

5. Middlebrook JL. Cell surface receptors for protein toxins. In: Simpson L (ed). Botulinum Neurotoxin and Tetanus Toxin. Cambridge, Academic Press, 1989: pp. 95-119.

6. Sarkarat F, Bohluli B, Kahali R. Officebased facial cosmetic procedures. In: Motamedi MHK (ed). A Textbook of Advanced Oral and Maxillofacial Surgery. London, IntechOpen, 2013: pp. 763-812.

7. Straube S, Derry S, Moore RA, Cole P.
Cervico-thoracic or lumbar sympathectomy for neuropathic pain and complex regional pain syndrome. Cochrane Database Syst Rev 2013; 9:1-20.

8. Romero FR, Haddad GR, Miot HA, Cataneo DC. Palmar hyperhidrosis: clinical, pathophysiological, diagnostic and therapeutic aspects. An Bras Dermatol 2016; 91:716-725.

9. Romero FR, Cataneo DC, Cataneo AJM. Outcome of percutaneous radiofrequency thoracic sympathectomy for palmar hyperhidrosis. Semin Thorac Cardiovasc Surg 2018; 30:362-366.

10. Garcia Franco CE, Perez-Cajaraville J, Guillen-Grima F, Espana A. Prospective study of percutaneous radiofrequency sympathicolysis in severe hyperhidrosis and facial blushing: Efficacy and safety findings. Eur J Cardiothorac Surg 2011; 40:e146-e151.

11. Purtuloglu T, Atim A, Deniz S, et al. Effect of radiofrequency ablation and comparison with surgical sympathectomy in palmar hyperhidrosis. Eur ] Cardiothorac Surg 2013; 43:e151-e154.

12. García-Barquín P, Beola JDA, Gracía JMB, et al. Percutaneous CT-guided sympathicolysis with radiofrequency for the treatment of palmar hyperhidrosis. J Vasc Interv Radiol 2017; 28:877-885.

13. Naumann M, Lowe N. Botulinum toxin type $\mathrm{A}$ in treatment of bilateral primary axillary hyperhidrosis: Randomised, parallel group, double blind, placebo controlled trial. BM] 2001; 323:596.

14. Lowe NJ, Yamauchi PS, Lask GP, Patnaik $\mathrm{R}$, lyer S. Efficacy and safety of botulinum toxin type $A$ in the treatment of palmar hyperhidrosis: A double-blind, randomized, placebo-controlled study. Dermatol Surg 2002; 28:822-827.

15. Lowe PL, Cerdan-Sanz S, Lowe NJ. Botulinum toxin type $A$ in the treatment of bilateral primary axillary hyperhidrosis: Efficacy and duration with repeated treatments. Dermatol Surg 2003; 29:545-548.

16. Heckmann M, Ceballos-Baumann AO, Plewig G. Botulinum toxin A for axillary hyperhidrosis (excessive sweating). N Engl J Med 2001; 344:488-493.

17. Lowe N, Campanati A, Bodokh I, et al. The place of botulinum toxin type $A$ in the treatment of focal hyperhidrosis. $\mathrm{Br}$ J Dermatol 2004; 151:1115-1122. 
